Supporting Information for

\title{
Quantification of Ni-N-O bond angles and NO activation by X-ray emission spectroscopy
}

Phan N. Phu, ${ }^{1+}$ Carlos Gutierrez, ${ }^{1}$ Subrata Kundu, ${ }^{2,3}$ Dimosthenis Sokaras, ${ }^{4}$ Thomas Kroll, ${ }^{4}$ Timothy H. Warren, ${ }^{2 *}$ S. Chantal E. Stieber ${ }^{1 *}$

${ }^{1}$ Department of Chemistry \& Biochemistry, California State Polytechnic University, Pomona, California 91768, United States.

2 Department of Chemistry, Georgetown University, Box 571227, Washington, DC 200571227, United States.

${ }^{3}$ School of Chemistry, Indian Institute of Science Education and Research Thiruvananthapuram, Kerala 695551, India.

${ }^{4}$ Stanford Synchrotron Radiation Lightsource, SLAC National Accelerator Laboratory, Menlo Park, California 94025, United States.

*Corresponding author email: sestieber@cpp.edu (S.C.E.S); thw@georgetown.edu (T.H.W.)

\section{Table of Contents}

1. XES Details $\quad$ S2

2. Computational Details $\quad$ S2 
1.

XES details
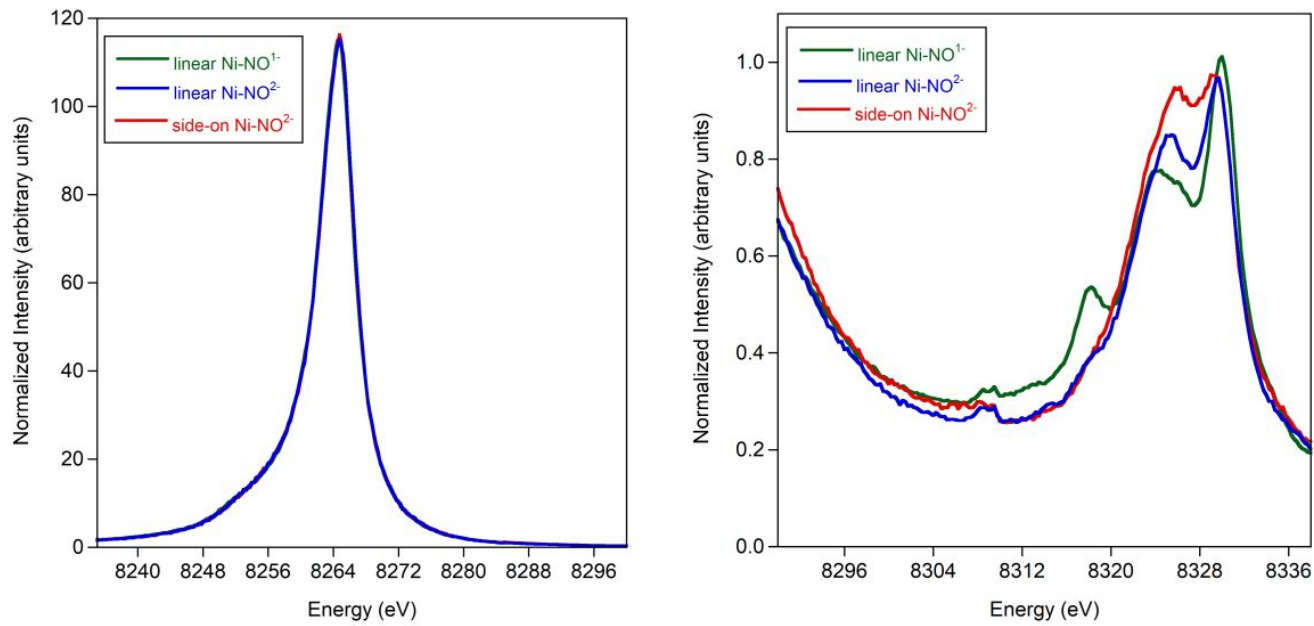

Figure S1. $\mathrm{K} \beta$ main line (left) and $\mathrm{VtC}$ (right) XES of [ $\left.{ }^{i} \mathrm{Pr}_{2} \mathrm{NN}_{\mathrm{F} 6}\right] \mathrm{NiNO}$ (linear $\mathrm{Ni}-\left(\mathrm{NO}^{1-}\right)$, green), $\left[{ }^{i} \operatorname{Pr}_{2} \mathrm{NN}_{\mathrm{F} 6}\right] \mathrm{Ni}\left(\mu-\eta^{1}: \eta^{1}-\mathrm{NO}\right) \mathrm{K}\left[2.2\right.$.2-cryptand] (linear $\mathrm{Ni}-\left(\mathrm{NO}^{2-}\right)$, blue), and [ $\left.{ }^{i} \mathrm{Pr}_{2} \mathrm{NN}_{\mathrm{F} 6}\right] \mathrm{Ni}\left(\mu-\eta^{2}: \eta^{2}-\right.$ $\mathrm{NO}) \mathrm{K}(18-\mathrm{crown}-6)(\mathrm{THF})$ (side-on $\mathrm{Ni}-\left(\mathrm{NO}^{2-}\right)$, red).

\section{Computational details}

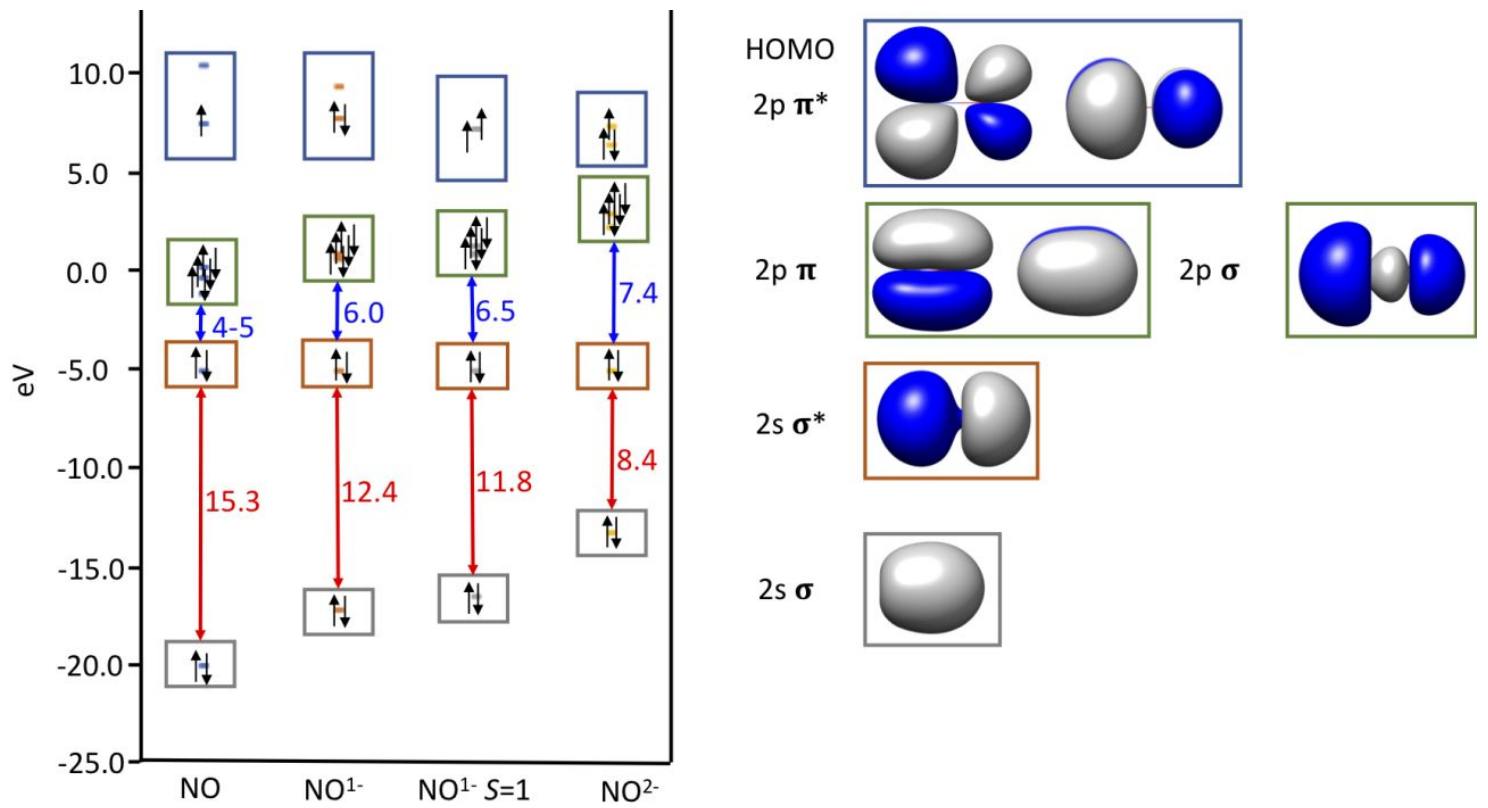

Figure S2. Qualitative $\mathrm{MO}$ energy diagram for $\mathrm{NO}, \mathrm{NO}^{1-}(\mathrm{S}=0), \mathrm{NO}^{1-}(\mathrm{S}=1)$, and $\mathrm{NO}^{2-}$ from DFT geometry optimization, along with visualization of corresponding orbitals. The energy scale was calibrated to the $2 \mathrm{~s} \sigma^{*}$ orbital to reflect the small change in energy of the NO $2 \mathrm{~s} \sigma^{*}$ orbital observed for the studied nickel nitrosyl complexes. 


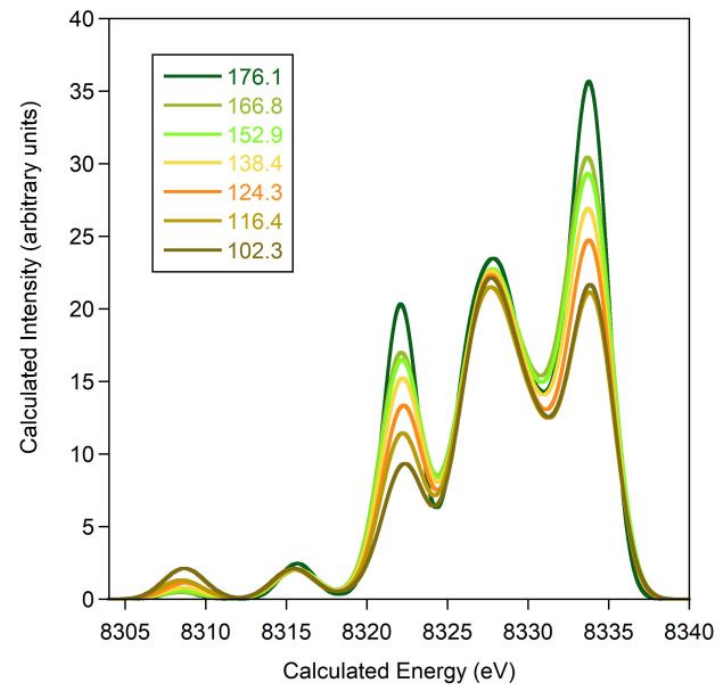

Figure S3. Calculated VtC XES spectrum of $\mathrm{Ni}-\left(\mathrm{NO}^{1-}\right)$ with varying the $\mathrm{Ni}-\mathrm{N}-\mathrm{O}$ angle from 102$176^{\circ}$. A shift of $217.0 \mathrm{eV}$ and a broadening of $2.5 \mathrm{eV}$ were applied to the computed data.
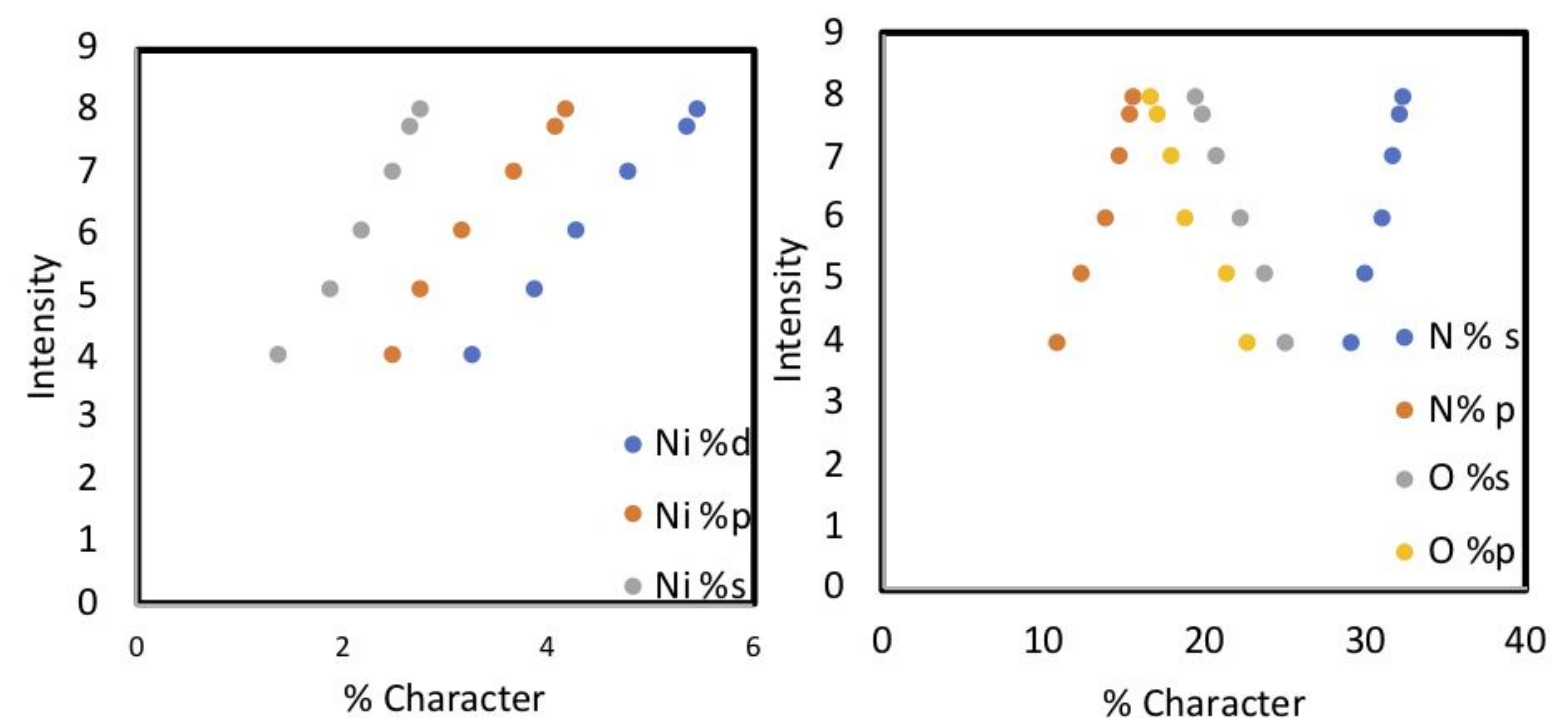

Figure S4. Total intensity versus calculated nickel character (left) and NO character (right) in transitions contributing to XES VtC spectral feature around $8322 \mathrm{eV}$ for the calculated VtC XES spectrum of $\mathrm{Ni}-\left(\mathrm{NO}^{1-}\right)$ with varying the $\mathrm{Ni}-\mathrm{N}-\mathrm{O}$ angle from $102-176^{\circ}$. 

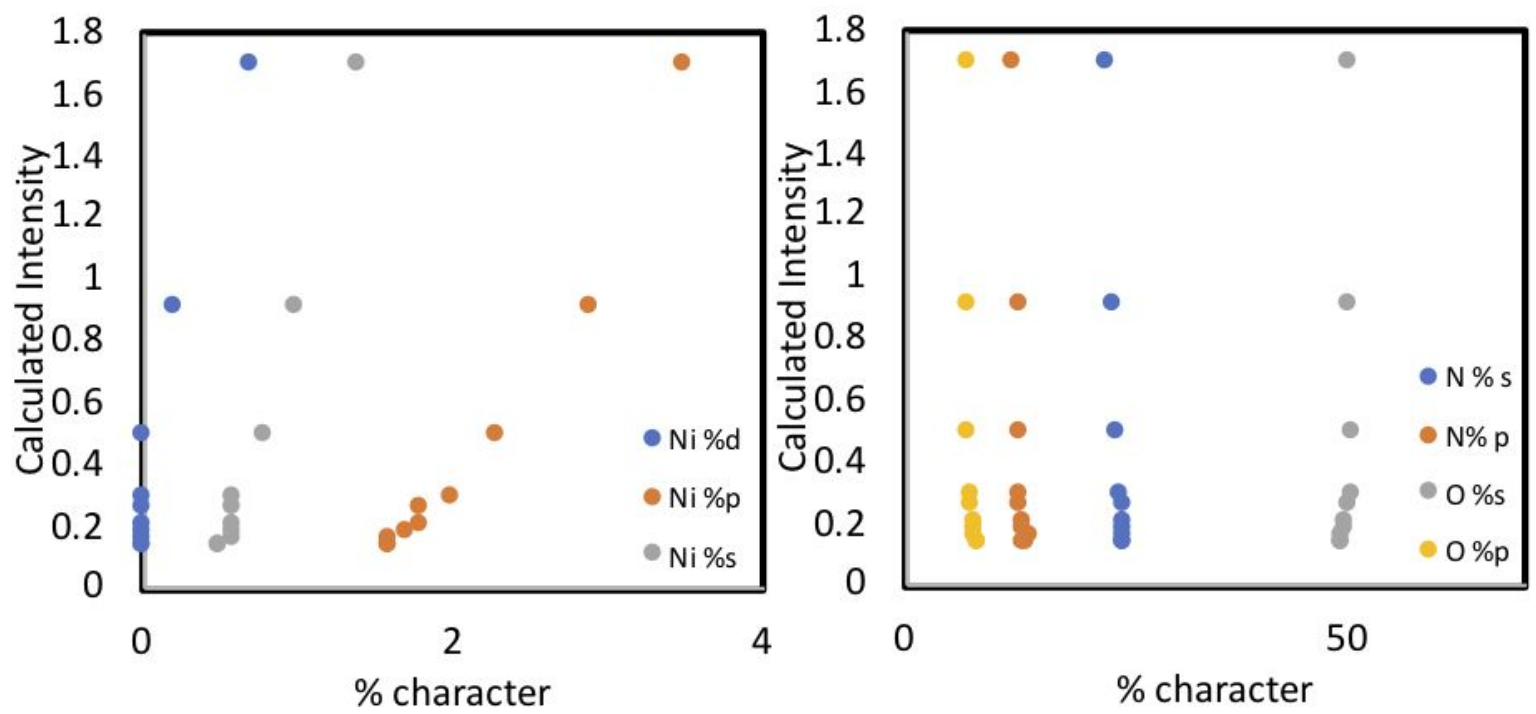

Figure S5. Total intensity versus calculated nickel character (left) and NO character (right) in transitions contributing to XES VtC spectral feature around $8310.7 \mathrm{eV}$ for the calculated VtC XES spectrum of $\mathrm{Ni}-\left(\mathrm{NO}^{2-}\right)$ with varying the $\mathrm{Ni}-\mathrm{N}-\mathrm{O}$ angle from $80-160^{\circ}$.
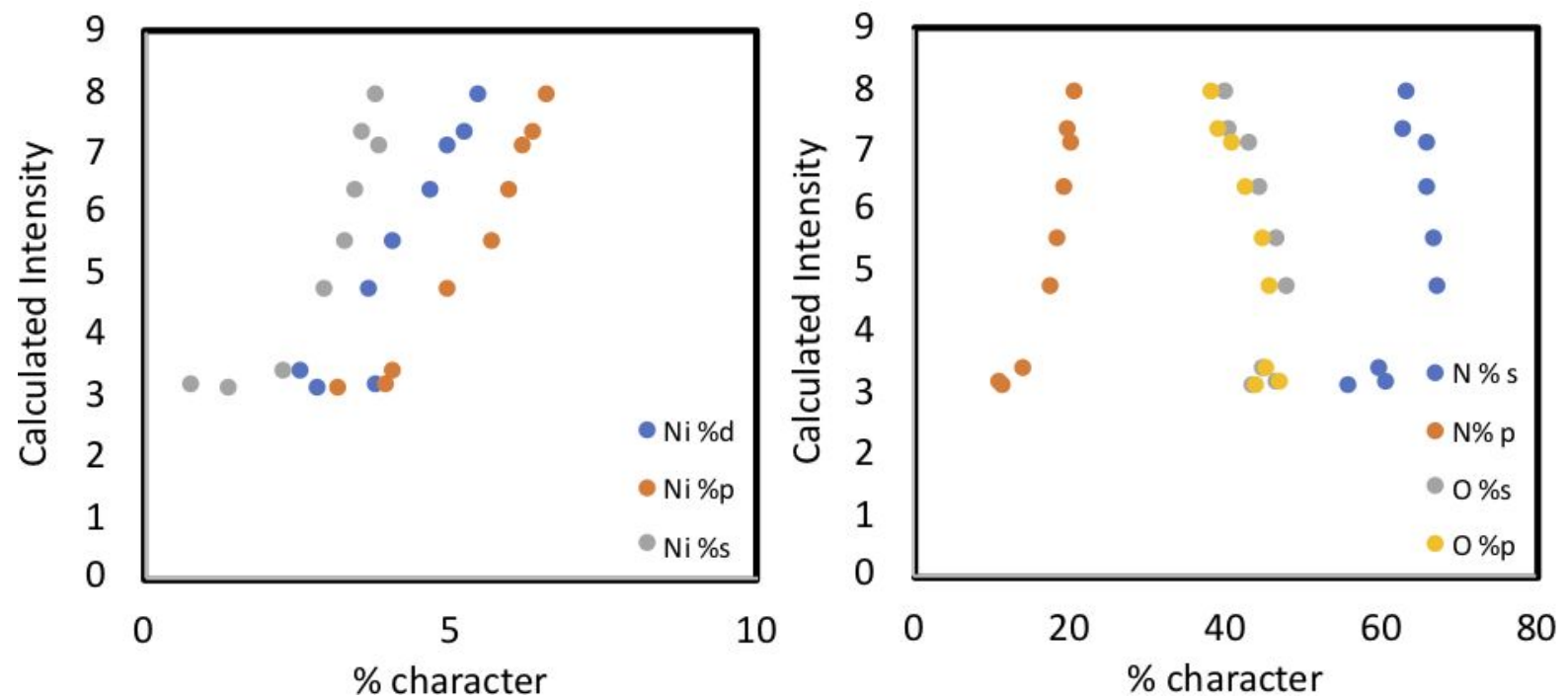

Figure S6. Total intensity versus calculated nickel character (left) and NO character (right) in transitions contributing to XES VtC spectral feature around $8322 \mathrm{eV}$ for the calculated VtC XES spectrum of $\mathrm{Ni}-\left(\mathrm{NO}^{2-}\right)$ with varying the $\mathrm{Ni}-\mathrm{N}-\mathrm{O}$ angle from $80-160^{\circ}$. 
Table S1. Ni-NO bond angles (deg.) and distances $(\AA \AA)$ resulting from geometry optimizations with fixed $\mathrm{Ni}-\mathrm{N}-\mathrm{O}$ bond angles for $\left[{ }^{i} \mathrm{Pr}_{2} \mathrm{NN}_{\mathrm{F} 6}\right] \mathrm{NiNO}, \mathrm{Ni}-\left(\mathrm{NO}^{1-}\right)$.

\begin{tabular}{|c|c|c|c|}
\hline Ni-N-O angle (deg.) & Ni-N distance $(\AA)$ & Ni-O distance $(\AA)$ & N-O distance $(\AA)$ \\
\hline 176.1 & 1.632 & 2.784 & 1.153 \\
\hline 166.8 & 1.635 & 2.772 & 1.155 \\
\hline 152.9 & 1.64 & 2.772 & 1.158 \\
\hline 138.4 & 1.654 & 2.639 & 1.163 \\
\hline 124.3 & 1.675 & 2.526 & 1.17 \\
\hline 116.4 & 1.701 & 2.457 & 1.173 \\
\hline 102.3 & 1.718 & 2.283 & 1.184 \\
\hline
\end{tabular}

Table S2. Ni-NO bond angle (deg.) and distances $(\AA ̊)$ resulting from geometry optimizations with fixed $\mathrm{Ni}-\mathrm{N}-\mathrm{O}$ bond angles for $\mathrm{Ni}-\left(\mathrm{NO}^{2-}\right)$.

\begin{tabular}{|c|c|c|c|}
\hline Ni-N-O angle (deg.) & Ni-N distance $(\AA)$ & Ni-O distance $(\AA)$ & N-O distance $(\AA)$ \\
\hline 160 & 1.771 & 2.944 & 1.217 \\
\hline 150 & 1.784 & 2.905 & 1.22 \\
\hline 140 & 1.801 & 2.848 & 1.222 \\
\hline 130 & 1.827 & 2.78 & 1.227 \\
\hline 120 & 1.849 & 2.688 & 1.234 \\
\hline 110 & 1.897 & 2.6 & 1.244 \\
\hline 100 & 1.877 & 2.427 & 1.247 \\
\hline 90 & 1.847 & 2.232 & 1.253 \\
\hline 80 & 1.83 & 2.036 & 1.265 \\
\hline
\end{tabular}
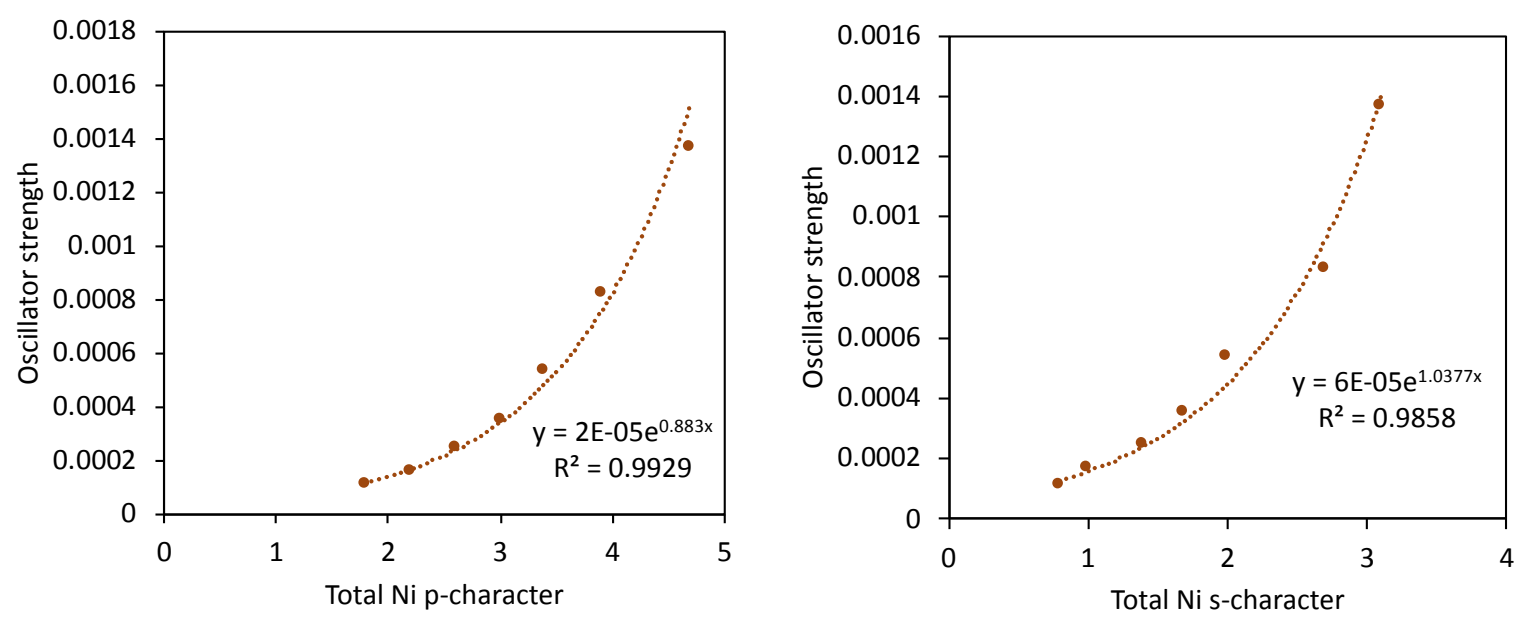

Figure S7. Total oscillator strength versus calculated nickel $p$-character (left) and s-character (right) in transitions contributing to XES VtC spectral feature around $8322 \mathrm{eV}$ for linear Ni( $\mathrm{NO}^{1-}$ ) with fixed $\mathrm{Ni}-\mathrm{N}$ distances. 
Table S3. Total oscillator strength versus calculated percent nickel $p$-character and $s$ character in transitions contributing to XES VtC spectral feature around $8322 \mathrm{eV}$ for linear $\mathrm{Ni}$ $\left(\mathrm{NO}^{1-}\right)$ with fixed $\mathrm{Ni}-\mathrm{N}$ distances.

\begin{tabular}{|c|c|c|c|}
\hline Energy (eV) & $\begin{array}{c}\text { Ni-N distance } \\
(\mathbf{\AA})\end{array}$ & $\begin{array}{c}\text { Total Ni } \boldsymbol{p} \text {-character } \\
\mathbf{( \% )}\end{array}$ & $\begin{array}{c}\text { Total Ni } \boldsymbol{s} \text {-character } \\
(\mathbf{\%})\end{array}$ \\
\hline 8321.99 & 1.527 & 4.7 & 3.1 \\
\hline 8322.07, & 1.627 & 3.9 & 2.7 \\
8322.20 & & 3.4 & 2.0 \\
\hline 8322.21 & 1.727 & 3.0 & 1.7 \\
\hline 8322.24 & 1.827 & 2.6 & 1.4 \\
\hline 8322.25, & 1.927 & 2.2 & 1.0 \\
8322.68 & & & 0.8 \\
\hline 8322.23, & 2.027 & 1.8 & \\
8322.57 & & & \\
\hline 8322.19, & 2.127 & & \\
8322.46 & & & \\
\hline
\end{tabular}

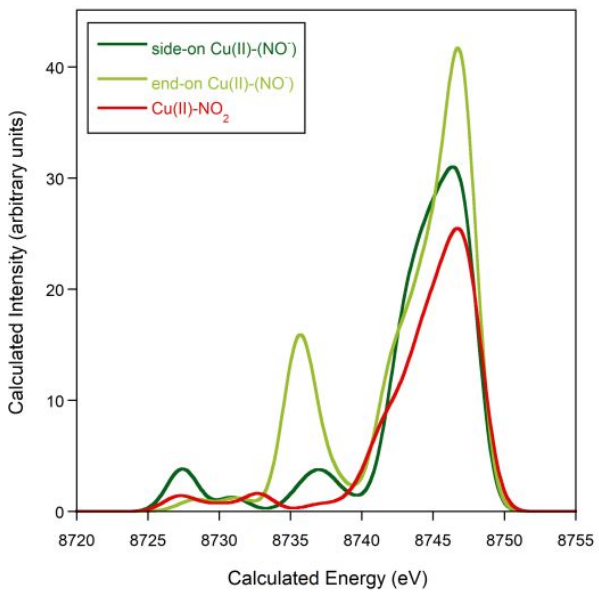

Figure S8. Calculated VtC XES spectrum of NiR for side-on $\mathrm{Cu}(\mathrm{II})-\left(\mathrm{NO}^{-}\right)$, end-on $\mathrm{Cu}(\mathrm{II})-\left(\mathrm{NO}^{-}\right)$, and $\mathrm{Cu}(\mathrm{II})-\mathrm{NO}_{2}$. The energy scale is not calibrated, and a broadening of $2.5 \mathrm{eV}$ was applied.
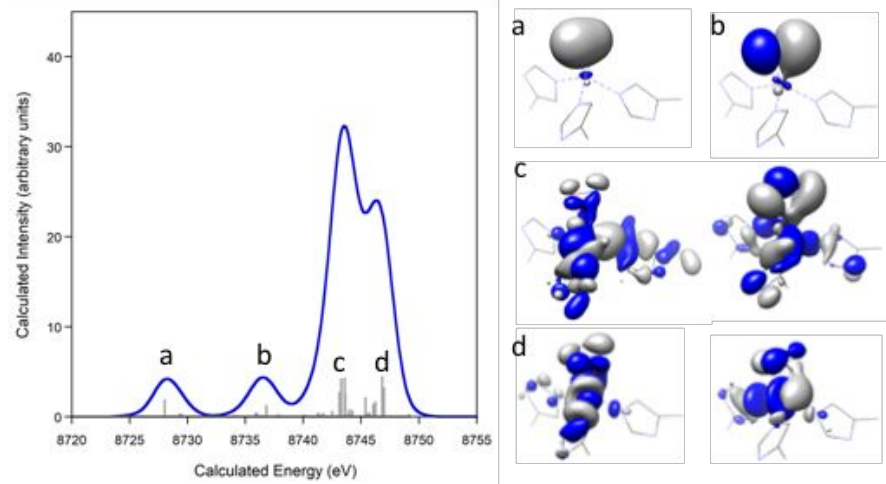

Figure S9. Calculated VtC XES spectrum of NiR for side-on $\mathrm{Cu}(\mathrm{I})-\left(\mathrm{NO}^{+}\right)$with the molecular orbitals that strongly contribute to the observed transitions. The energy scale is not calibrated, and a broadening of $2.5 \mathrm{eV}$ was applied. 

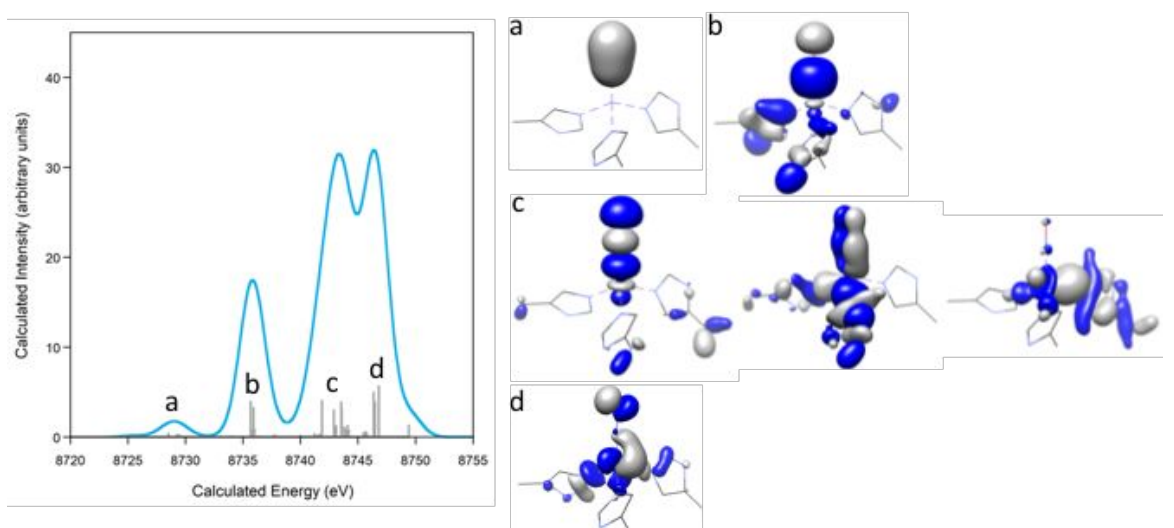

Figure S10. Calculated VtC XES spectrum of NiR for end-on $\mathrm{Cu}(\mathrm{I})-\left(\mathrm{NO}^{+}\right)$, with the molecular orbitals that strongly contribute to the observed transitions. The energy scale is not calibrated, and a broadening of $2.5 \mathrm{eV}$ was applied.

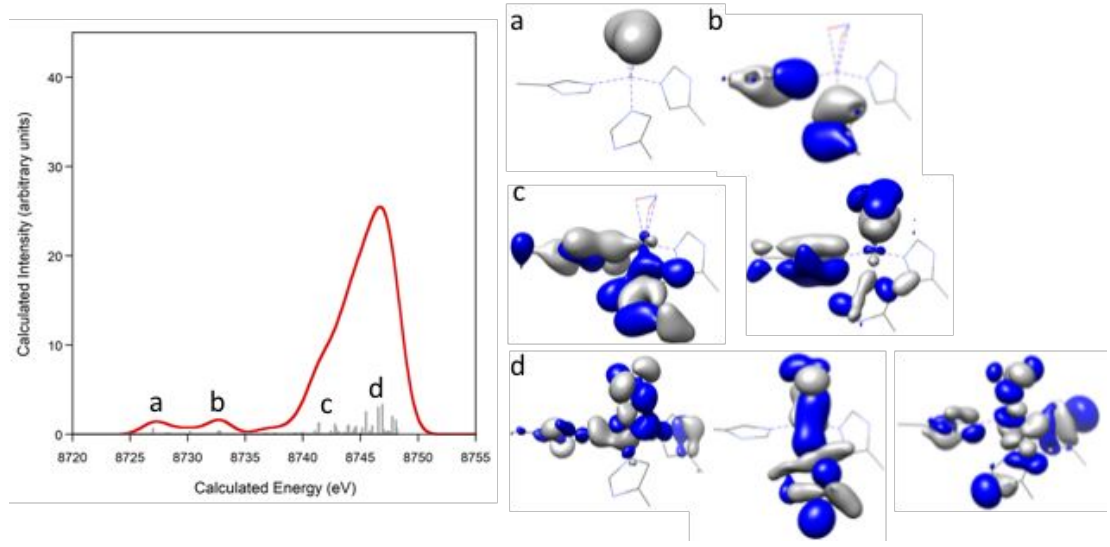

Figure S11. Calculated VtC XES spectrum of NiR for $\mathrm{Cu}(\mathrm{II})-\mathrm{NO}_{2}$ with the molecular orbitals that strongly contribute to the observed transitions. The energy scale is not calibrated, and a broadening of $2.5 \mathrm{eV}$ was applied.

Representative geometry optimization input file for fixed Ni-N-O angle of $150^{\circ}$ (ORCA):

! UKS B3LYP RIJCOSX SlowConv TightSCF def2-SV(P) def2-SVP/J Normalprint OPT PAL8 \%geom Constraints

$\{$ A $024150.0 \mathrm{C}\}$

end

end

\%basis NewGTO 28 "def2-TZVP(-f)" end

NewGTO 7 "def2-TZVP(-f)" end

NewGTO 8 "def2-TZVP(-f)" end

NewAuxGTO 28 "def2-TZVP/J" end

NewAuxGTO 7 "def2-TZVP/J" end 
NewAuxGTO 8 "def2-TZVP/J" end

end

\%scf Maxiter 3000

TolE 1E-7

TolErr 1E-6

end

* xyz 01 coordinates.xyz

\section{Representative XES input file (ORCA):}

! UKS BP86 TZVP def2-TZV/J TightSCF SlowConv COSMO SCFConv7 PAL8

! Normalprint

! grid4 nofinalgrid

\%basis newgto Ni "CP(PPP)" end

end

\%method SpecialGridAtoms 28

SpecialGridIntAcc 7

end

\%scf Maxlter 1000

TolE 1e-7

TolErr 1e-6

end

* xyzfile 01 coordinates.xyz

\%xes
CoreOrb 0,0
OrbOp 0,1
End

\section{Atomic coordinates used in calculations (xyz format):}

1. Previously published geometry optimized coordinates for linear $\mathrm{Ni}^{-\mathrm{NO}^{1-}}{ }^{-}$, linear $\mathrm{Ni}-\mathrm{NO}^{2-}$, and side-on $\mathrm{Ni}^{-\mathrm{NO}^{2-}}$ were used from reference 3.

2. All other geometry optimized coordinates and atomic coordinates can be found on the Zenodo repository under DOI: 10.5281/zenodo.3911324 